\title{
Estratégia de saúde da família e idoso com demência: o cuidado pelos profissionais de saúde
}

\author{
Family health strategy and older adults with dementia: \\ care provided by health professionals
}

Hellen Guedes do Nascimento (https://orcid.org/0000-0002-5019-3555) ${ }^{1}$

Ana Elisa Bastos Figueiredo (https://orcid.org/0000-0001-7207-0911) ${ }^{1}$

${ }^{1}$ Departamento de Estudos sobre Violência e Saúde Jorge Careli, Escola Nacional de Saúde Pública, Sérgio Fiocruz. Av. Brasil 4036/700, Manguinhos. 21040-361 Rio de Janeiro RJ Brasil. hellenguedes.psi@

hotmail.com

\begin{abstract}
This is a qualitative, descriptive-analytical study developed in a primary health care facility organized by the ESF. Semi-structured interviews were conducted with five health professionals: two doctors, a nurse, and two community health workers (ACS). The interview's guiding topics were respondent data (name, gender, age, professional category, and seniority in the service); elderly care; care to relatives; perception of the experience of extreme situations in the elderly monitoring; and challenges in the ESF in assisting older adults. Representations related to ESF professionals are directly related to the position held. The ACS are appointed as the facility's thermometer and eyes. Doctors perceive a great demand and see themselves as biomedical knowledge holders and medication prescribers. Nursing is perceived as a professional mediator between ACS and doctors. The walk-in demands of older adults and their families continue to be sustained by the tripod doctor, equipment (tests and procedures), and medication. As family hardships comprise dementia, the perception of care as a burden, and the socioeconomic constraints are intensifying aspects of dementia. The creation of a bond between professionals, relatives, and older adults was perceived as an emotional health care device fundamental for monitoring dementia cases.
\end{abstract}

Key words Older adults with dementia, Care, Health professionals/ESF
Resumo Estudo qualitativo descritivo-analítico desenvolvido em um setor de atenção primária à saúde organizada pela Estratégia de Saúde da Familia (ESF). Realizadas entrevistas semiestruturadas com cinco profissionais de saúde: dois médicos, uma enfermeira e dois agentes comunitários de saúde (ACS). Tópicos norteadores da entrevista: dados sobre o entrevistado; atendimento ao idoso; atenção aos familiares; percepção acerca de vivência de situações-limite no acompanhamento do idoso; e dificuldades encontradas na ESF para a assistência do idoso. Representações acerca dos profissionais da ESF estão diretamente relacionadas ao cargo exercido. Os ACS são descritos como o termômetro e os olhos da unidade. Os médicos se percebem com uma demanda grande e como detentores do saber biomédico e prescritores de medicação. A enfermagem como o profissional mediador entre ACS e médico. As demandas espontâneas dos idosos e familiares continuam sustentadas no tripé: médico, equipamentos e medicamentos. As dificuldades do familiar em compreender a demência, a percepção do cuidado como um peso e as limitações socioeconômicas foram aspectos descritos como intensificadores do processo demencial. A criação de vínculo entre os profissionais, familiares e idosos foi percebida como um dispositivo emocional de cuidado em saúde fundamental para o acompanhamento dos casos de demência.

Palavras-chave Idoso com demência, Cuidado, Profissionais de saúde, Estratégia de saúde da família 


\section{Introdução}

O Brasil encontra-se em um processo de transição epidemiológica pela queda da mortalidade e da fecundidade a partir de mudanças socioeconômicas positivas ${ }^{1}$. Neste contexto, ocorreu a inversão da pirâmide populacional com a redução da quantidade de crianças e o aumento do número de idosos. Analisando o processo de envelhecimento da população, o último Censo de 2010, realizado pelo Instituto Brasileiro de Geografia e Estatística ${ }^{2}$ demonstrou que os idosos já representavam 10,8\% da população. Este aumento da expectativa de vida tem gerado repercussões sociais, econômicas, políticas, culturais e na situação de saúde da população brasileira.

Em termos de saúde podemos enfatizar o aumento de casos de doenças crônico-degenerativas e o acometimento de traumas decorrentes de causas externas, como violência e acidentes ${ }^{3}$. Dentre as doenças crônicas-degenerativas, encontra-se a demência que é uma doença associada ao processo de envelhecimento. A demência poderia ser definida como uma progressiva alteração da memória e de pelo menos uma outra função cognitiva, representando declínio em relação ao nível de funcionamento prévio, com impacto no comportamento e na intensidade suficiente para interferir no aspecto pessoal, social e profissional ${ }^{4}$. Em estágios mais avançados os comprometimentos acarretam a dependência total.

A demência afeta significativamente a família do idoso, principalmente o familiar cuidador, aquele que se torna responsável por toda rotina de cuidado em famílias que escolhem cuidar do idoso ou que não podem arcar com os custos para terceirizar este cuidado. A doença provoca mudanças objetivas e subjetivas na dinâmica familiar que podem ser difíceis de lidar, podendo gerar conflitos e desagregações na estrutura familiar. A situação de dependência é agravada pela vulnerabilidade social de muitas famílias dos idosos brasileiros. As dificuldades de sobrevivência acabam transformando o adoecimento desse idoso numa experiência ainda mais difícil de suportar. O cuidado do idoso no processo demencial constitui-se como um desafio para os profissionais de saúde que lidam com este adoecimento, tendo em vista que a dinâmica familiar, os sintomas apresentados e a sobrecarga no cuidado demarcam a heterogeneidade de cada caso de demência ${ }^{5}$.

O cuidado, de acordo com Bauman ${ }^{6}$, está relacionado à uma "atitude emocional" que surge como resistência à objetificação do ser por promover relações de parcerias entre os indivíduos e não relações que os tornam objetos a serem usados. Emocional, portanto, são todos os atos e pensamentos que não se limitam a avaliações ou medições. O modelo de atenção à saúde hegemônico encontra-se na contramão da perspectiva de Bauman sobre o cuidado. Tal modelo reproduz a objetificação, valorizando os procedimentos e os exames em detrimento da escuta, do acolhimento e do vínculo. Estas atitudes, dentre outras, evidenciam a produção do distanciamento emocional entre o profissional de saúde e aquele que necessita da assistência. Pinheiro ${ }^{7}$ afirma que o cuidado em saúde não se restringe apenas ao desempenho das competências e tarefas técnicas de atenção, tem a ver com as interações positivas entre usuários, profissionais e instituições. Incluem o envolvimento, o relacionamento, o acolhimento, a escuta do sujeito, o respeito ao sujeito e a sua história de vida.

O distanciamento emocional e a objetificação do ser humano que está sendo cuidado pode favorecer situações que correspondem ao avesso do cuidado, como a violência. Minayo ${ }^{3}$ descreve 3 formas nas quais a violência contra o idoso se apresenta: 1) estrutural, decorrente da desigualdade social e naturalizada nas manifestações de pobreza, miséria e discriminação; 2) interpessoal, que ocorre nas interações e nas relações cotidianas entre as pessoas; 3 ) institucional, que acontece a partir de uma aplicação ou omissão na gestão de políticas públicas pelo Estado e pelas instituições de assistência, reproduzindo relações assimétricas de poder, de domínio, menosprezo e de discriminação.

No que diz respeito à saúde pública, a Estratégia da Saúde da Família (ESF) no Brasil, enquanto modelo de atenção primária, tem como objetivo promover o cuidado integral dos indivíduos e da coletividade, devendo acolher todas as demandas de saúde e de sofrimento que surgem em seu território8. . A hipótese deste estudo é que o modelo ESF é um dispositivo de saúde pública fundamental para intervir e cuidar das questões do idoso demenciado e de seus familiares cuidadores por vislumbrar o "indivíduo em relação", em contraposição ao "indivíduo biológico" como cita Merhy'.

A ESF leva em consideração o território e as relações socioafetivas e como porta de entrada do sistema de saúde recebe a demanda de idosos decorrentes do processo de envelhecimento da população brasileira. Este estudo tem como objetivo compreender a partir da percepção dos 
profissionais de saúde atuantes na ESF, como o idoso demenciado e o familiar cuidador são percebidos, recebidos e assistidos por este serviço diante da complexidade envolvida no cuidado dessa população.

\section{Desenho metodológico}

A pesquisa qualitativa foi escolhida por permitir uma compreensão das percepções e das crenças dos sujeitos, fazendo com que se desvelem a história, os sentidos, os significados e as ideologias envolvidas nesta forma de ver o mundo. Minayo ${ }^{10}$ afirma que esta modalidade de pesquisa faz com que o dizer revele as condições estruturais, os sistemas de valor, as normas e os símbolos. O entrevistado atua como porta-voz das representações de determinados grupos em determinada condição histórica, socioeconômica e cultural. O uso da pesquisa qualitativa proporciona uma compreensão aprofundada ao considerar aspectos humanos singulares e relacionais que escapam da avaliação e da quantificação epidemiológica e estatística, mas que é tão relevante quanto estes métodos na compreensão da vida, em estudos e em pesquisas.

O estudo qualitativo descritivo-analítico foi desenvolvido em uma unidade de atenção primária à saúde organizada pela ESF, situada na zona norte do município do Rio de Janeiro. A unidade atende em torno de 14.000 usuários, possui oito equipes de saúde da família composta por 83 profissionais de saúde, como enfermeiros, médicos e agentes comunitários de saúde, composta também por outros profissionais de saúde bucal, vigilância sanitária, farmácia e também a gerente da clínica.

Para contemplar o objetivo do estudo foram realizadas entrevistas semiestruturadas que permitem uma relação mais simétrica entre entrevistador e entrevistado, favorecendo a exposição de sentimentos, percepções e expectativas, desvelando a subjetividade deste sujeito e permitindo uma análise de acordo com a perspectiva do entrevistado. As entrevistas tiveram os seguintes tópicos norteadores para as questões formuladas: dados sobre o entrevistado (nome, sexo, idade, categoria profissional e tempo de serviço); o atendimento ao idoso com demência na ESF; a atenção aos familiares cuidadores; a percepção acerca de vivência de situações-limite no acompanhamento do idoso demenciado; e as dificuldades encontradas na ESF para a assistência do idoso demenciado.
Este estudo é parte dos resultados de uma dissertação de mestrado, a pesquisa foi submetida ao Comitê de Ética da Secretaria Municipal de Saúde do Rio de Janeiro e ao da Escola Nacional de Saúde Pública, e atendeu as normas da resolução nº466/12 do CNS. Os entrevistados assinaram Termo de Consentimento Livre e Esclarecido. Os nomes utilizados neste estudo são fictícios para manter o anonimato. Após o aceite do comitê de ética restaram sete meses para a realização da pesquisa de campo e a análise dos dados. Sendo assim, foram entrevistados cinco profissionais de saúde: dois médicos, uma enfermeira e dois agentes comunitários de saúde (ACS), que faziam parte de equipes distintas dentro da unidade de saúde pesquisada.

Os profissionais selecionados para participar da pesquisa atenderam aos seguintes critérios: ter nível superior, médio ou fundamental, atuando diretamente com o usuário e com vínculo de trabalho de pelo menos seis meses na unidade. Foram excluídos estagiários e profissionais que não tinham contato direto com usuários do serviço de saúde. As entrevistas foram gravadas e transcritas para a realização da análise do discurso (AD).

A AD trata o conteúdo coletado nas entrevistas como um processo de significação, transcendendo a ideia de que a fala do entrevistado teria o objetivo de transmitir uma informação. O discurso é compreendido como um objeto que funde a língua, o sujeito e a história. A AD se inicia com o exercício de desnaturalizar a relação palavra-coisa ${ }^{11}$, compreendendo que o sentido da palavra não nasce com ela e que não existe apenas um modo de dizer sobre algo, transformando assim a língua em objeto discursivo. Em seguida, tornam-se visíveis os eixos conceituais que aparecem ao longo do discurso dos entrevistados. Diante destes eixos conceituais se desvelaram os núcleos significantes a partir da singularidade de cada fala, gesto e não dito ${ }^{12}$.

\section{Resultados e discussão}

\section{Os profissionais de saúde entrevistados}

João - 32 anos, é baiano, formado em medicina desde 2015 com experiência anterior em atenção secundária e terciária em saúde. É residente do programa de medicina da família e comunidades desde 2016.

Laura - 32 anos, é carioca, formada em medicina desde 2010 com experiência anterior na 
atenção primária, secundária e terciária em saúde. É residente do programa de medicina da família e comunidades da unidade desde 2016.

Daniela- 25 anos, é carioca, formada em enfermagem desde 2013 com experiência anterior na coordenação da atenção primária. É enfermeira contratada por CLT desde 2015.

Maurício - 58 anos, é carioca, reside no bairro da unidade desde que nasceu e é ACS desde a sua inauguração em 2012, foi um dos pioneiros segundo ele. Esta foi sua primeira experiência de trabalho na área da saúde.

Letícia - 41 anos, é carioca, reside no bairro desde que nasceu e é ACS. Trabalhou durante 10 anos em administração de hospital público na região antes de ingressar na unidade em 2012. Conta que foi uma das 30 primeiras agentes da unidade.

\section{O Discurso dos profissionais de saúde}

No processo de AD foi elaborado um mosaico significante constituído pelos eixos conceituais identificados no discurso dos profissionais de saúde e os núcleos significantes desvelados nas falas sobre cada eixo conceitual.

A Figura 1 é um Mosaico Significante: Análise do discurso dos profissionais de saúde.

\section{Eixo Conceitual - Os profissionais de saúde da ESF}

\section{Núcleos significantes: atuação dos agentes comunitários de saúde, médicos e enfermeiros}

A equipe multiprofissional da ESF deve ser composta por, no mínimo, médico generalista ou especialista em saúde da família ou médico de família e comunidades, enfermeiro generalista ou especialista em saúde da família, auxiliar ou técnico de enfermagem e $\mathrm{ACS}^{8}$.

De acordo com Letícia ACS, a atuação do ACS é descrita como fundamental para a realização do modelo da ESF. É o olhar do médico, do enfermeiro no território, Letícia e Maurício ACS, por estarem presentes em maior frequência nas casas e circulando no território, além de serem pessoas que fazem parte da história da comunidade. A enfermeira e os médicos entrevistados enalteceram o trabalho do ACS durante a entrevista descrevendo-os como peça chave, termômetro.

A vertente de trabalho dos ACS para a enfermeira e os médicos se relaciona com territorialização. De acordo com a médica Laura, são eles que determinam se o território tem muita grávida ou muitos idosos, produzem números e estatísticas que são fundamentais para definir a estratégia de

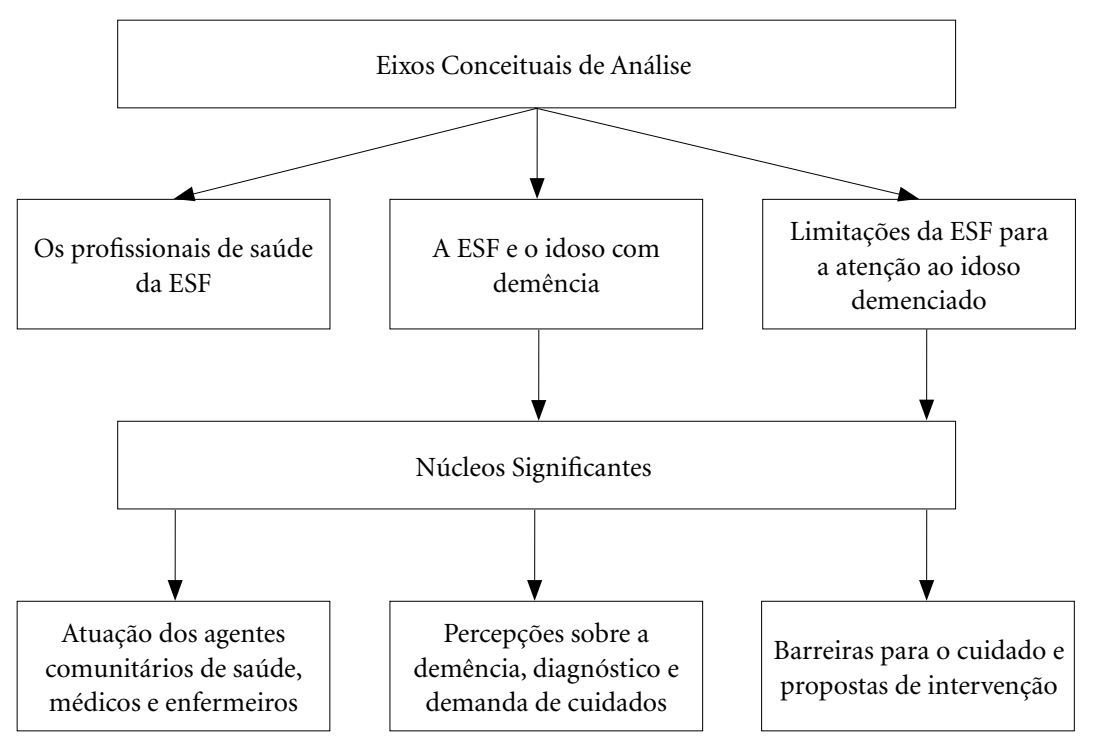

Figura 1. Mosaico Significante. 
trabalho. Ou seja, eles conhecem a comunidade em que atuam e favorecem o vínculo da mesma com a unidade de saúde. A territorialização é um processo que tem sido preconizado em diversas iniciativas do SUS, no entanto, em diversos momentos é reduzida a formatação do espaço, extensão geométrica determinada. É preciso considerar que além de uma extensão geométrica, há um perfil demográfico, epidemiológico, administrativo, tecnológico, político, social e cultural, e está em constante construção ${ }^{13}$.

Os ACS são reconhecidos nas formações discursivas dos médicos e enfermeiras como agentes fundamentais na identificação das questões da família do idoso demenciado. Para Laura médica, o agente sinaliza: doutora ou enfermeira, tem alguma coisa de errado com a dona $M$. (paciente atendida pela unidade de saúde) Vamos lá fazer uma visita?

Esta atuação é revestida de grande responsabilidade para os ACS, por serem exigidos a terem um olhar clínico para notar as dificuldades iniciais, intermediárias e tardias de um processo demencial, e perceber e manejar as mudanças no contexto familiar do idoso com demência. Os ACSs, Letícia e Maurício, relatam que não possuem capacitação para este tipo de avaliação e observação clínica.

A médica Laura ressalta em seu discurso: $o$ sucesso não é o mesmo se não tiver uma coisa do agente de saúde de muita dedicação [...] se o agente não for dedicado ele deixa passar muitas situações. Esta fala transmite a noção da responsabilização dos ACSs na ESF, sendo os erros da equipe associados ao trabalho dos ACSs, situação que pode gerar tensão e sobrecarga para estes profissionais.

O discurso de Maurício traduz a percepção do lugar do próprio ACS e revela uma sobrecarga na rotina de trabalho: nós deveríamos tomar conta de 600 pessoas para que nós não ficássemos doentes. Eu já cheguei a ter 1.057, 900 e pouco, a minha base é essa, 800 e pouco, 900 e pouco, 980... Eu nunca tive uma ouvidoria até hoje e já tenho até presente, é inevitável.

Apesar da percepção da sobrecarga pelo quantitativo da população assistida que pode atrapalhar seu rendimento no trabalho e fazê-lo adoecer, Maurício se envaidece pela criação do vínculo com a comunidade e por ser reconhecido pelos usuários - estes sentimentos surgem como estratégia de defesa para lidar com a experiência de sobrecarga.

O cuidado em saúde demanda disponibilidade de tempo para construir e manter a atenção às famílias. Além disso, exige a resistência ao mode- lo de saúde que tem foco e uso excessivo de procedimentos e exames no lugar da escuta e do acolhimento. A representação da atuação dos ACSs no discurso dos médicos e da enfermeira reflete uma mudança de paradigma entre os profissionais de saúde na ESF ao vislumbrar o indivíduo em relação $o^{9}$ na comunidade, na sua família e com os profissionais da unidade de saúde.

Nas formações discursivas dos médicos e ACSs, a atuação da enfermagem foi compreendida como aquela que vai mais às visitas domiciliares e que acompanha mais de perto os ACSs. $O$ enfermeiro me passa muita informação básica, de acordo com o médico João. A percepção da enfermeira Daniela sobre sua atuação é diferente do observado pelos médicos e ACS: $O$ médico focando ali na parte biomédica dele, a gente no cuidado de enfermagem tenta focar um projeto terapêutico singular [...] tenta atender de uma forma holistica de acordo com as necessidades dele (usuário). A atuação da enfermagem neste discurso reflete uma intermediação ligando o saber biomédico do médico e o vínculo do ACS na comunidade. O sentido atrelado ao trabalho da enfermagem como o detentor do olhar da clínica ampliada descaracteriza a corresponsabilidade de todos os profissionais de saúde da ESF diante do Projeto Terapêutico Singular. A gestão do cuidado deve ser coproduzida e cogerida entre todos os profissionais da saúde da família.

Os médicos Laura e João são residentes, permanecem dois anos no mesmo território, na mesma unidade de saúde e constroem vínculo com equipe, usuários e comunidade. Quando termina a residência, eles são desligados da comunidade rompendo o vínculo, o que compromete a continuidade do trabalho e a relação terapeuta-paciente. Situação diferente dos profissionais contratados, como ACS e enfermeiros, que se estabelecem na comunidade por mais tempo e possuem maior oportunidade de construção de vínculo.

O médico João ponderou: médico da família não trata só o idoso. Nesse caso, João refere-se ao vasto leque de demanda para o atendimento, desde gestantes e crianças até idosos, e também o número de pacientes sob a responsabilidade da equipe de saúde a que pertence, que gira em torno de 5.000. A fala do médico João sinaliza que existem demandas múltiplas, o que implica dificuldade em lidar com situações específicas como a demência. Esse fato pode contribuir para o subdiagnóstico de demência, pois os médicos são responsáveis pelo diagnóstico dos usuários do serviço. A questão do subdiagnóstico estudada 
por diversos autores ${ }^{14-19}$ envolve baixa qualidade no registro e rastreio da demência; dificuldades nos diagnósticos diferenciais; características próprias da prática do serviço; e atitude do médico na triagem dos casos também podem influenciar no subdiagnóstico.

O médico João relata: o médico está habilitado a reconhecer e fazer o diagnóstico, mas existem alguns diagnósticos que a gente precisa da neurologia, demonstrando a necessidade de uma rede de cuidados de referência e contrarreferência. A médica Laura sintetiza o cuidado do idoso demenciado pelo médico de família: evitar a consequência da consequência, ou seja, lidar com a prevenção secundária e terciária, com as comorbidades decorrentes dos sintomas e do processo de envelhecimento. Laura assinala a necessidade de uma intervenção mais direta e pontual e a necessidade de educação da família para evitar confrontos e acidentes. Enfatiza em seu discurso: ESF é cuidado para todos, não só para os idosos.

Na perspectiva da ACS Letícia: a médica vê que precisa de uma medicação, ela transcreve a medicação que foi prescrita pelo outro médico (especialista), uma percepção da prática médica associada à prescrição e transcrição de medicamentos. O pouco tempo que o residente se estabelece no território e a grande demanda de usuários são fatores que podem impactar nas limitações e na qualidade do cuidado.

Outra questão relatada pelos profissionais foi a inexistência do Núcleo de Apoio à Saúde da Família (NASF) na unidade pesquisada. A questão do apoio aos profissionais de saúde foi também tratada em pesquisas ${ }^{20,21}$, ao mostrarem que seria um fator facilitador para transformar a prática individualizada do médico de família em uma prática mais abrangente, sistemática e interprofissional, para um maior reconhecimento dos programas e serviços disponíveis e para o encaminhamento e o reforço da detecção precoce da demencia. A equipe do NASF deveria integrar psicólogos, educador físico, terapeutas ocupacionais, fisioterapeutas, fonoaudiólogos, médico geriatra, psiquiatra, dentre outros ${ }^{8}$. Os profissionais não souberam explicar o motivo de não poder contar com esses profissionais para as demandas que poderiam ser acolhidas por esta equipe.

\section{Eixo Conceitual - A ESF e o idoso com demência}

\section{Núcleos Significantes: percepções sobre a demência, diagnóstico e demanda de cuidados}

A demência é uma questão de saúde públi$\mathrm{ca}^{22}$. Inicialmente é caracterizada por um sofrimento psicológico intenso diante da noção da perda da consciência de si. O avançar da doença e as dificuldades cognitivas de memória, raciocínio e linguagem torna mais difícil para o idoso falar sobre seu estado emocional interno. Diante da progressão da doença, as manifestações neuropsiquiátricas surgem como agravante ${ }^{23}$.

A médica Laura destaca os cuidados paliativos como forma de atenção na demência. A perspectiva paliativa acaba sentenciado o idoso a partir do seu diagnóstico, pois o idoso deixa de receber cuidados e estimulações que, além de retardar o avanço da doença, contribuem para melhorar aspectos emocionais e relacionais. Dança de salão, taekondo, artesanato e academia da terceira idade são atividades desenvolvidas na ESF, mas os idosos com demência não são incluídos.

A enfermeira Daniela explica que a classificação da doença não deve ultrapassar o ser humano que está por trás da doença, e em seguida discorre: será que eu estou falando para o vento? Traduzindo a sensação de estranhamento ao lidar inicialmente com o idoso demenciado que acaba produzindo ideias preconcebidas acerca das potencialidades e possibilidades do idoso, que inclusive em estágios moderados com auxílio do cuidador pode participar e se estimular em diversas atividades.

O ACS Maurício associa os casos de demência à dificuldade de ficar aguardando e ir até a unidade de saúde, o que é uma realidade nos casos avançados, ressalta a relevância do cuidado domiciliar nestes casos. De acordo com a ACS Letícia, a demência ainda é compreendida como preocupação da atenção secundária e terciária: geralmente já tomam medicações, já foram no neurologista, acompanham em algum outro local, mas como nós somos só a parte de clínica geral, eles ficam aqui. A entrevistada tem um histórico pregresso de trabalho em hospitais, em sua compreensão persiste uma visão hospitalocêntrica e a demência como restrita ao fator biológico. Essa percepção limitada acerca das possibilidades da atuação da ESF nestes casos, minimiza as potencialidades do serviço diante do idoso com demência e seus familiares. A demência é uma doença que vai além do acometimento biológico, 
afeta o idoso emocionalmente, repercute consideravelmente na dinâmica familiar e, principalmente, no familiar cuidador que assume os cuidados do idoso ${ }^{23}$.

A médica Laura ressalta que na ESF: não precisa encaminhar porque a ESF tem o NASF e $o$ matriciamento para dar suporte, no entanto a enfermeira Daniela ressalta que não existe equipe do NASF dando suporte à unidade. A dinâmica de trabalho da unidade nos casos de demência parece conflitante, vejamos: o ACS Maurício relata que é realizado o encaminhamento para as especialidades nos casos de demência, enquanto a médica Laura sustenta suas colocações teóricas sobre o suporte do NASF e matriciamento nos casos complexos e a enfermeira Daniela traz a questão prática da fragilidade da unidade pela ausência do serviço do NASF. Lembra ainda que se o objetivo da ESF, como atenção básica, é diminuir a necessidade do idoso permanecer sendo atendido em serviços de saúde de alta complexidade, a presença do NASF poderia contemplar esse objetivo e ressalta a necessidade da multidisciplinaridade na atenção primária, além do médico, do enfermeiro e do agente comunitário de saúde.

Importante salientar que ao longo do discurso dos profissionais não foram relatadas situações em que fosse utilizada a rede de atenção psicossocial ou outras redes de suporte de saúde e suporte assistencial para a realização de um cuidado integral. Os encaminhamentos citados foram relacionados a especialidades de saúde que tratam da demanda biológica da doença, como a neurologia.

A médica Laura, numa perspectiva diagnóstica, considera que os sintomas podem ser percebidos a partir de três vias: queixa do usuário idoso, do familiar e percepção do próprio profissional de saúde ao acompanhar longitudinalmente a familia, reconhecendo as mudanças e as falas típicas do início do processo demencial. O médico João descreve que a demanda surge: na queixa de que o paciente tá esquecendo alguma coisa, que tá vendo mudanças no paciente, ou que acha que é teatro do paciente. Os médicos entrevistados citaram a aplicação de testes de rastreio Mini-Exame do Estado Mental - MEEM para avaliação diagnóstica em caso de suspeita de demência. O médico João: no percurso diagnóstico e ao longo do cuidado gosto de encaminhar o paciente para neurologia para avaliação. Teipel et al. ${ }^{24}$ mostraram a importância de se utilizar teste para avaliação de sintomas em pacientes com demência como: Inventário Neuropsiquiátrico (NPI), MEEM para cognição global, QoL-AD para qualidade de vida, escala de Utilização de Recursos na Demência (RUD) para custos na demência e escala para avaliar estresse do cuidador.

O discurso da ACS Letícia exemplifica como surgem as demandas espontâneas dos usuários: me procuram para falar: olha, o meu pai foi lá no neurologista e ele pediu uma tomografia. Referese, portanto, à demanda por exames, inclusive daqueles que frequentam outros serviços ambulatoriais da saúde suplementar ou para buscar a medicação que neurologistas prescreveram e que são fornecidos pela unidade de saúde. A ESF ainda é percebida de acordo com o modelo clássico de assistência a doenças em suas demandas espontâneas, corroborando com a crítica de Sousa e Hammann ${ }^{25}$, quando afirmam que as ações de saúde continuam sustentadas no tripé: médico, equipamentos (exames e procedimentos) e medicamentos, e não conseguem mudar a lógica organizativa dos serviços e dos sistemas de saúde.

\section{Eixo Conceitual - Limitações da ESF para a atenção ao idoso demenciado}

\section{Núcleos significantes: barreiras para o cuidado e propostas de intervenção}

Em termos de barreiras para o atendimento do idoso com demência e seus familiares na ESF, a ACS Letícia relata que a equipe é reduzida, principalmente por ter poucos residentes de medicina para realizarem as visitas domiciliares. Neste mesmo sentido, o médico João ressalta a agenda cheia do médico [...] quantitativo de pacientes com outros diagnósticos acaba infelizmente sendo mais importantes do que a demência. João relata que em diversas situações há necessidade de desmarcar visitas domiciliares para os idosos demenciados em função de doenças mais agudas, como gestante de alto risco.

A enfermeira Daniela entende como barreira as dificuldades relacionadas à humanização face a uma rotina de trabalho intensa com indicadores e metas mensais de atendimentos a serem cumpridas pelos profissionais. Percebe que, por um lado, é bom existirem metas para direcionar o caminho a ser seguido em cada linha de cuidado, mas por outro, que é ruim pela pressão para cumprir a meta e não perder o emprego. Diante desta condição, o trabalho termina tendo como foco as demandas imediatas dos pacientes: $A h$, é uma receita que você precisa? Então, toma.

O ACS Maurício destaca a problemática que o idoso enfrenta quando é encaminhado para tratamentos como a fisioterapia em locais distantes, 
pela falta de uma rede de cuidados próxima e por não ter condições de se locomover de ônibus ou por longos trajetos, além de muitas vezes não dispor de recursos financeiros para tal.

O médico João encontra dificuldades na parceria com o cuidador: às vezes você tenta manter contato com o familiar e isso leva um ano. Ele relata situações nas visitas domiciliares em que na sua concepção não chega a considerar negligência, mas percebe que muitos familiares enxergam como peso o cuidado do idoso. A grande demanda de atendimento e a falta de equipe multiprofissional se esbarra com questões de saúde da família do idoso, que necessitam de acompanhamento psicossocial para serem apoiados, orientados e cuidados, prevenindo situações de violência contra o idoso. Minayo ${ }^{3}$ ressalta que no seio familiar a violência é de difícil diagnóstico, os idoso muitas vezes negam e de acordo com as denúncias, negligência, abuso psicológico e abuso financeiro e patrimonial são os mais ocorridos.

Os ACSs por estarem presentes na comunidade e na visita domiciliar percebem as mudanças estabelecidas na dinâmica familiar. Segundo Maurício alguns idosos: às vezes ficam agressivos, às vezes porque não querem tomar um banho, $e$ (o familiar) tem que contornar aquela situação; é muito difícil para aquele que está cuidando. As dificuldades de compreender o processo demencial, muitas vezes por falta de instrução, e a limitação socioeconômica da família são descritos pela médica Laura como barreiras para a realização de um cuidado adequado.

$O$ ato de cuidar aos poucos se torna uma atividade complexa. Aparecem sentimentos difíceis de manejar, acarreta privação e modificação do estilo de vida do cuidador. A doença segue um percurso que vai além do evento biológico propriamente dito, evidenciando construções psicológicas e socioculturais permeadas de significados e interpretações por parte dos que vivenciam e dos que mantêm relações de cuidado com o idoso ${ }^{26}$. As construções subjetivas acerca do cuidado impactam diretamente na qualidade da relação estabelecida entre cuidador e cuidado; associar o cuidado como um peso torna a experiência algo difícil de suportar.

Neste sentido os entrevistados trouxeram em seu discurso a importância do vínculo entre os profissionais de saúde e os idosos e seus familiares cuidadores. O médico João relata: quando um paciente cria um vínculo com você, adere ao tratamento muito melhor; quando ele gosta do profissional se sente mal até em não fazer o tratamento [...] ele acredita em você, ele sabe que você está ali para ajudá-lo. Cecílio $^{27}$ sobre o cuidado aponta a competência técnica, a postura ética profissional e a capacidade de construir vínculos como os principais elementos na atuação do profissional de saúde.

A enfermeira Daniela destaca a dificuldade de construir vínculo com o idoso demenciado que tem alterações cognitivas que impedem a comunicação entre profissional e paciente. Percebe como estratégia a manutenção do vínculo também com os familiares cuidadores, se aproximando mais do paciente, já que os familiares conseguem interpretar seus comportamentos e identificar suas necessidades: Você vê sim que é produtivo, vocêfalar, você vê que por mais que eles esqueçam, eles têm uma referência sua.

O cuidado dos familiares cuidadores é tão primordial quanto o do idoso demenciado no cuidado da saúde da família. A ACS Letícia destaca sempre oferto vaga no atendimento para os familiares do idoso, convoco para grupo de hipertensos e diabéticos, vejo a família como um todo. Alguns entrevistados perceberam o cuidado do cuidador como o suporte de conscientização para motivar o tratamento do idoso com demência evitando assim uma piora do quadro. A médica Laura percebe que alguns cuidadores têm dificuldades para cuidar de si, e que: tentar conscientizar a família de que precisa haver um cuidado individual, precisa de um asseio individual, é um trabalho muito árduo, porque geralmente quem tem um idoso na família vive para isso. A médica revela este meu olhar atual é muito especializado, que não é o olhar que tinha há três, uma mudança de perspectiva a partir do seu ingresso na saúde da família.

Pesquisas internacionais ${ }^{28-30}$ apresentaram barreiras para o cuidado integral coincidentes com as colocações dos entrevistados e outras que nos pareceram importantes mencionar: problemas na comunicação com especialistas em saúde mental, limitações no tempo, falta de familiaridade com os recursos da comunidade e a dificuldade dos profissionais em lidar com mudanças. Alguns autores ${ }^{28,31}$ também sinalizaram a necessidade de uma assistência domiciliar em casos de dificuldade de deslocamento. A articulação de um modelo de cuidado mais holístico foi apresentada por Apesoa-Varano et al. ${ }^{32}$, assim como a carga emocional e as questões jurídicas envolvidas no cuidado do idoso com demência.

Diante destas barreiras, alguns entrevistados propuseram como soluções: a redução da quantidade de pacientes atendidos por cada equipe de saúde da família, para 1.500 usuários, pois poderiam coordenar melhor o acolhimento e 
fortalecer o vínculo; a participação de outras especialidades, como: a fisioterapia e a psicologia na equipe multidisciplinar da unidade básica de saúde da família para a realização de visitas domiciliares.

\section{Considerações finais}

O estudo demonstrou que as representações acerca dos profissionais da ESF estão diretamente relacionadas ao cargo exercido pelo profissional. Os profissionais descreveram o ACS como o termômetro e os olhos da unidade. Os médicos se percebem com uma demanda grande e são vistos como detentores do saber biomédico e prescritores de medicação. A enfermagem é vista como o profissional mediador entre ACS e médico.

As significações acerca do processo demencial refletem os níveis mais avançados da doença e os cuidados realizados são vistos como paliativos. No que se refere ao diagnóstico, os médicos entrevistados citaram o teste de rastreio minimental, a atenção as queixas do idoso, do familiar, a percepção do próprio profissional de saúde ao acompanhar a família e os médicos destacaram a importância da rede de referência com neurologia.

As demandas espontâneas dos idosos e familiares continuam sustentadas no tripé: médico, equipamentos (exames e procedimentos) e medicamentos. As dificuldades do familiar cuidador em compreender a demência, a percepção do cuidado como um peso e as limitações socioeconômicas foram aspectos descritos como intensificadores do processo demencial. A criação de vínculo entre os profissionais, familiares cuidadores e idosos, foi percebido como um dispositivo emocional de cuidado em saúde fundamental para o acompanhamento dos casos de demência. Alguns profissionais percebem que os familiares cuidadores abdicam de si na medida em que cuidam do outro; outros compreendem a atenção ao familiar cuidador no sentido de motivá-lo para manter o cuidado do idoso.

A grande demanda de atendimento e a prioridade dada a situações agudas, a ausência dos profissionais de apoio, como psicólogos e fisioterapeutas, as dificuldades na percepção da singularidade e na construção do vínculo diante foram percebidas como barreiras no cuidado na demência. Outro ponto percebido foi o não estabelecimento de uma rede de cuidados com outros serviços públicos, privados ou comunitários.

O modelo de atenção à saúde da família em sua teoria é um dispositivo de saúde pública com possibilidade de intervenção e cuidado integral para questões do idoso demenciado e de seus familiares por vislumbrar o "indivíduo em relação", em contraposição ao "indivíduo biológico" como cita Merhy ${ }^{9}$, no entanto a prática do serviço analisada neste estudo demonstra que modelo biomédico hegemônico permanece ditando as regras no processo de trabalho da ESF. Grande demanda de atendimentos por equipe e o foco no tripé medicação, exames e médico, ausência do suporte do NASF, da rede de atenção psicossocial e toda a possibilidade de formação de rede comunitárias de cuidado, acabam limitando o cuidado e proporcionando medidas paliativas de cuidado.

\section{Colaboradores}

HG Nascimento e AEB Figueiredo participaram de toda a elaboração do artigo. 


\section{Referências}

1. Chaimowicz F. Epidemiologia e o envelhecimento populacional no Brasil. In: Freitas E, organizador. Tratado de geriatria e gerontologia. $2^{\text {a }}$ ed. Rio de Janeiro: Guanabara Koogan; 2006. p.106-130.

2. Instituto Brasileiro de Geografia e Estatística (IBGE). Sinopse do Senso Demográfico de 2010. Rio de Janeiro: IBGE; 2011.

3. Minayo M. Manual de enfrentamento à violência contra a pessoa idosa. É possivel prevenir. É necessário superar. Brasília: Secretaria de Direitos Humanos da Presidência da República; 2014

4. Bertolucci P. Demências. In: Ortiz K. Z. Distúrbiosneurológicos adquiridos: linguagem e cognição. Barueri: Manole; 2005. p. 295-312.

5. Ferretti C, Bottino C. Intervenções Psicossociais em Cuidadores e Familiares de Pacientes com Demência. In: Bottino C, Laks J, Blay SL, organizadores. Demências e transtornos cognitivos em idosos. Brasil: Editora Guanabara Koogan; 2006. p. 371-376.

6. Bauman Z. Vida em fragmentos: sobre a ética pós moderna. Rio de Janeiro: Editora Zahar; 2011

7. Pinheiro R. Cuidado em saúde. Verbetes. Dicionário da Educação Profissional em Saúde. Rio de Janeiro: Fiocruz; 2009.

8. Brasil. Ministério da Saúde (MS). Política Nacional de Atenção Básica. Brasília: MS; 2012.

9. Merhy E. O ato de cuidar: A alma dos serviços de saúde. Rev SUS Brasil: Cadernos de textos 2004; 2004:108137.

10. Minayo M. O desafio do conhecimento: Pesquisa qualitativa em saúde. São Paulo: Hucitec Editora; 2014.

11. Orlandi E. Análise do Discurso: princípios e procedimentos. Campinas: Pontes Editora; 2009.

12. Orlandi E. Discurso e Texto: formulação e circulação dos sentidos. Campinas: Pontes Editores; 2012

13. Monken M, Barcellos C. Vigilância em saúde e território utilizado: possibilidades teóricas e metodológicas. Cad Saude Publica 2005; 21(3):898-906.

14. Connolly A, Gaehl E, Martin H, Morris J, Purandare $\mathrm{N}$. Underdiagnosis of dementia in primary care: Variations in the observed prevalence and comparisons to the expected prevalence. Aging Ment Health 2011; 15(8):978-984.

15. Del canto de hoyos-alonso M, Bonis J, Bryant V, Alcalá M, Puime A. Quality of registration of dementia diagnosis in primarycare: The situation in Spain in 20022011. Atencion Primaria 2016; 48(1):33-41

16. Eichler T, Thyrian J, Hertel J, Kohler L, Wucheler D, Teipel S. Rates of Formal Diagnosis in People Screened Positive for Dementia in Primary Care: Results of the DelpHi-Trial. J Alzheimers Dis 2014; 42(2):451458.

17. Boise L. Eckstrom E, Fagnan L, King A, Golbald M, Buckley D, Morris C. The Rural Older Adult Memory (ROAM) study: A practice-based intervention to improve dementia screeningand diagnosis. Journal of the American Board of Family Medicine 2010; 23 (4):486498.

18. Fowler N, Harrawood A, Frame A, Perkins A, Gao S, Boustani $\mathrm{M}$. The Indiana University Cognitive Health Outcomes Investigation of the Comparative Effectiveness of dementia screening (CHOICE) study: Study protocol for a randomized controlled trial. Trials 2014; 15(1):209.
19. Olazaran J. Torrero P, Cruz I, Aparicio E, Sanz A, Begué $\mathrm{C}$. Mild cognitive impairment and dementia in primary care: the value of medical history. Fam Pract 2011; 28(4):385-392.

20. Lee L, Hillier L, Stolee P, Heckman G, Gagnon M, Harvey D. Enhancing Dementia Care: A Primary Care-Based Memory Clinic. J Am Geriatr Soc 2010; 58(11):2197-2204.

21. Callahan C, Boustani M, Weiner M., Beck R., Livin L, Hendrie H. Implementing dementia care models in primary care settings: The Aging Brain Care Medical Home. Aging Ment Health 2011; 15(1):5-12.

22. Organização mundial de saúde (OMS). Demência: Prioridade da Saúde Pública. Genebra: OMS; 2012

23. Caramelli P, Bottino C. Tratando os sintomas comportamentais e psicológicos na demência. J Bras Psiquiatr 2007; 56(2):83-87.

24. Teipel S, Thyrian J, Hertel J, Eichler T, Wucherer D, Hoffmann W. Neuropsychiatric symptoms in people screened positive for dementia in primary care. Int Psychogeriatr 2015; 27(1):39-48.

25. Sousa M, Hamann E. Programa Saúde da Família no Brasil: uma agenda incompleta? Cien Saude Colet 2009; 14(Supl. 1):1325-1335.

26. Oliveira A, Caldana R. As Repercussões do Cuidado na Vida do Cuidador Familiar do Idoso com Demência de Alzheimer. Revista Saúde e Sociedade 2012; 21(3):675-685.

27. Cecílio L. Apontamentos teórico-conceituais sobre processos avaliativos considerando as múltiplas dimensões da gestão do cuidado em saúde. Interface (Botucatu) 2011; 15(37):589-99.

28. Callahan MC, Boustani AM, Shimid AA, Austrom MG, Miller D, Gao S, Hendrie H. Alzheimer's disease multiple intervention trial (ADMIT): Study protocol for a randomized controlled clinical trial. Trials 2012; 13:92

29. Fortinsky RH, Zlateva I, Delanei C, Kleppinger A. Primary CarePhysicians' Dementia Care Practices: Evidence of Geographic Variation. Gerontologist 2010; 50(2):179-191

30. Franz CE, Barker JC, Kim K, Flores Y, Jenkins C, Kravitz R, Hinton, L. When help becomes a hindrance: Mental health referral systems as barriers to care for primary care physicians treating patients with alzheimer's disease. Am J Geriatr Psychiatry 2010; 18(7):576-585.

31. Thume E, Facchini L, Tomasi E, Azambuja L, Vieira S. Assistência domiciliar a idosos: fatores associados, características do acesso e do cuidado. Rev Saude Publica 2010; 44(6):1102-1111.

32. Apesoa-varano E, Barker J, Hinton L. Curing and Caring: The Work of Primary Care Physicians With Dementia Patients. Qualit Health Res 2011; 21(11):14691483.

Artigo apresentado em 14/07/2020

Aprovado em 19/11/2020

Versão final apresentada em 21/11/2020

Editores chefes: Maria Cecília de Souza Minayo, Romeu Gomes, Antônio Augusto Moura da Silva. Editora Associada da área de Saúde do Idoso: Joselia Oliveira Araújo Firmo 